\section{Seeds of no change}

Apomixis - the development of an embryo in the absence of meiosis and fertilization - occurs naturally in some wild species of plant but has been difficult to engineer in major crop species, in which it would be the ideal way to conserve the traits of superior hybrid genotypes. Two new studies describe promising routes to achieving apomixis in Arabidopsis thaliana and maize, respectively, by using genetic mutants.

In A. thaliana the first step in apomixis - obtaining diploid gametes - can be carried out by using mutant plants (such as MiMe and dyad) that produce diploid gametes owing to defects in meiosis. To allow the diploid gamete to form a parthogenetic, diploid embryo, Marimuthu, Jolivet, Ravi and colleagues fertilized the female diploid gamete with male gametes in which chromosomes had been engineered to be eliminated after fertilization (by mutation in the centromere-specific histone CENH3). Approximately one-third of the seeds produced in crosses between a female diploid gamete and a male gamete containing the genome elimination mutation were diploid, and almost all of these embryos were clones of the maternal genome and retained the heterozygosity of the mother, as would be desirable for practical applications. By repeating the process with the female gamete of the cloned F1 plants, the authors showed that clonal propagation could occur for more than one generation.

Rather than use existing mutant lines, which produce low numbers of viable gametes $(\mathrm{MiMe}$ is an

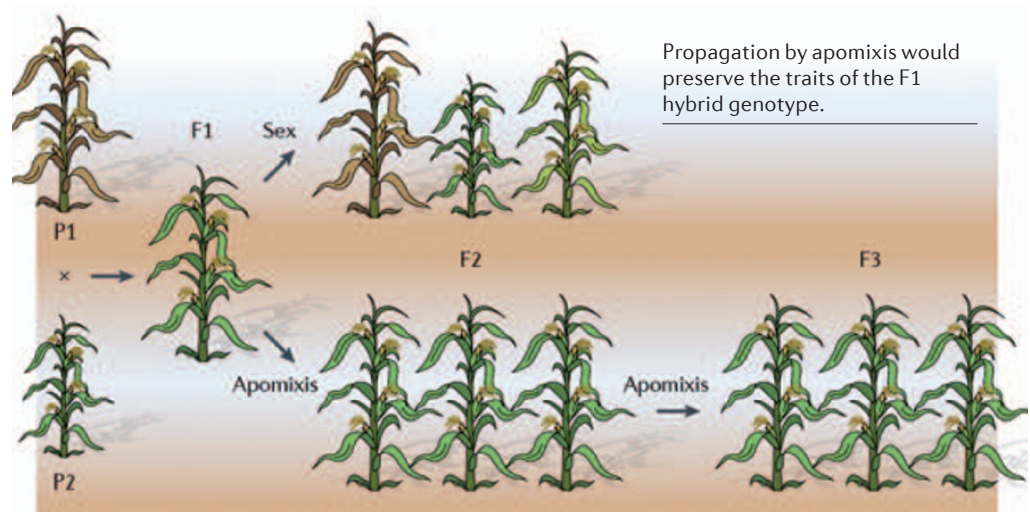

exception), Singh and colleagues set out to screen maize for new dominant mutations that lead to diploid gametes by disrupting the reduction step in meiosis. The authors identified a mutation with such a property: the mutation, which affects the ARGONAUTE protein AGO104, causes defective chromatin condensation during meiosis and results in a lack of chromosome segregation. AGO104 appears to be a functional orthologue of $A$. thaliana AGO9, in that it acts non-cell-autonomously to regulate transcription specifically in the ovule. AGO104 is also required for methylation at many non-CG sites and so would seem to contribute genetically and epigenetically to sexual development. Intriguingly, the study suggests small RNA pathways as a new route to engineering apomixis.

These studies highlight new strategies for cloning plants through seeds. The practical implementation of apomixis in agriculture, however, would require the apomictic plant to self-propagate, without the help of a second, crossing line.

Tanita Casci

ORIGINAL RESEARCH PAPERS

Marimuthu, M. P. A. et al. Synthetic clonal reproduction through seeds. Science 331, 876 (2011)|Singh, M. et al. Production of viable gametes without meiosis in maize deficient for an ARGONAUTE protein. Plant Cell 15 Feb 2011 (doi:10.1105/tpc.110.079020) 